\title{
Non-Lyapunov Stability of the Fractional-Order Time-Varying Delay Systems
}

\author{
Mirko Mišljen ${ }^{1)}$ \\ Mihailo Lazarević ${ }^{2)}$
}

\begin{abstract}
In this paper, the finite-time stability criteria are extended to nonlinear nonhomogeneous perturbed fractional-order systems including multiple time-varying delays. The sufficient conditions of a stability for the fractional systems with multiple time delays are obtained by using the generalized and classical Gronwall's approach. A numerical example is presented to illustrate the validity of the obtained result.
\end{abstract}

Key words: continuous system, nonlinear system, time-delay system, time delay, system stability, non-Lyapunov stability, finite-time stability, fractional order system.

\section{Introduction}

$\mathrm{I}_{\mathrm{e}}^{\mathrm{N}}$ recent years, fractional differential equations are extensively studied $[1,2]$. The existence of solutions of the fractional differential equations is studied in [1]. The existence and uniqueness of solutions of the linear fractional differential equations for the fractional timedelay systems is considered in [2]. Time delays are present in various engineering systems, such as long transmission lines, hydraulic, pneumatic, and electric networks, chemical processes, etc. Time-delay systems are described by differential-difference equations. This type of equations belongs to the class of functional differential equations [3].

Stability is an important issue in the system and control theory. Stability of time-delay systems has been investigated over the last few decades [4]. Stability analysis of time-delay systems is more complicated than stability analysis of the systems without time delays because timedelay systems include the derivative of the time-delayed state. The existence of pure time delay, regardless if it is present in the state or/and control, may cause an undesirable system transient response, or generally, even an instability [5].

In the stability analysis of time-delay systems, two approaches have been adopted [5]. One approach involves the stability conditions that do not include information on the delay, and in the other approach, the stability conditions take into account information on the delay. The first approach is called the delay-independent criteria and generally provides simple algebraic conditions. Because there is no upper limit to time delay, the delay-independent criteria are often regarded as conservative in practice, where the unbounded delays are not realistic.

The largest number of stability conditions for time-delay systems deal with linear models. Both necessary and sufficient conditions have been developed for some special cases, which are mainly delay-dependent. In many papers, the stability criteria are presented by using the Lyapunov's second method and the concept of matrix measure [6,7].

Various concepts of stability, such as finite-time stability, practical stability, robust stability, internal stability, external stability, have been studied for fractionalorder systems in [8-18]. Finite-time and practical stability is considered in the papers [8-14]. Robust stability results for the linear fractional systems are presented in [15]. Matignon [16] studied the internal stability and external stability (bounded input-bounded output (BIBO) stability) of linear fractional systems. Stability analysis of the linear fractional systems with multiple delays is discussed in [17]. Analytical stability bound for the fractional delayed systems by using the Lambert function is investigated by Chen and Moore [18].

The stability of the fractional-order systems cannot be analyzed by using the algebraic criteria that are developed for stability analysis of integer-order systems, such as the Hurwitz criterion, since the fractional systems do not have characteristic polynomial. Instead, the fractional systems have pseudopolynomial with a rational power-multivalued function. The Lyapunov methods have been developed for the analysis of stability of the linear and nonlinear integer systems and have been extended to the analysis of stability of the fractional systems.

On the other side, there are only few papers that consider the non-Lyapunov stability (finite-time and practical stability) of the fractional systems. Recently, for the first time, the finite-time stability of the fractional delay systems is reported in [19]. Using the recently obtained generalized Gronwall inequality [20], the stability test procedure for the linear nonhomogeneous fractional systems with a constant time delay is suggested in the paper [21].

Besides, there are also many systems with multiple time

\footnotetext{
1) Military Technical Institute (VTI), Ratka Resanovića 1, 11132 Belgrade, SERBIA

2) University of Belgrade, Faculty of Mechanical Engineering, Department of Mechanics, Kraljice Marije 16, 11120 Belgrade 35, SERBIA

Correspondence to: Mirko Mišljen; e-mail: mirko0705@gmail.com
} 
delays in the practical applications. In that way, it is more necessary to study systems with multiple time delays than those with a single delay. Recently, some works are devoted to finite-time stability issues for the fractionalorder neural networks with delays [22,23].

This paper presents the system stability from the nonLyapunov point of view. The finite-time stability for the class of nonlinear nonhomogeneous perturbed fractional systems including multiple time-varying delays is proposed using generalized Gronwall inequality and then by using classical Bellman-Gronwall inequality [10].

\section{Fractional Calculus Definitions}

The idea of a fractional calculus has been known since the development of a classical calculus [24].

The fractional calculus deals with differential and integral operators of non-integer order. The fractional differentiation and integration is an extension and generalization of the conventional integer-order differentiation and integration. Over the last few decades, the applications of fractional calculus had a considerable progress [25]. For example, wide and fruitful applications can be found in rheology, viscoelasticity, acoustics, optics, chemical and statistical physics, robotics, control theory, electrical and mechanical engineering, bioengineering, etc. [26-29]. The main reason for the success of fractional calculus applications is that these new fractional-order models are often more accurate than integer-order ones, i.e. there are more degrees of freedom in the fractional-order model than in the corresponding classical one [30]. All fractional operators consider the entire history of the process being considered, thus being able to model the nonlocal and distributed effects often encountered in natural and technical phenomena [28-31].

The fractional derivative and integral may be defined in many ways [25-28]. The definitions that are mainly used are the Riemann-Liouville definition, the GrünwaldLetnikov definition, and the Caputo definition.

Definition 1. Let $f(\cdot) \in \mathcal{C}[a, b]$ be a continuous function over the finite interval $[a, b]$. The RiemannLiouville fractional derivative of the order $\alpha \in \mathbb{C}$, $\operatorname{Re} \alpha \geq 0, n-1 \leq \operatorname{Re} \alpha<n, n \in \mathbb{N}$, is defined as [25]:

$$
\begin{gathered}
\mathrm{RL}^{\alpha} \mathrm{D}_{a, t} f(t)=\frac{1}{\Gamma(n-\alpha)}\left(\frac{\mathrm{d}}{\mathrm{d} t}\right)^{n} \int_{a}^{t} \frac{f(s)}{(t-s)^{\alpha-n+1}} \mathrm{~d} s, \\
t \in[a, b],
\end{gathered}
$$

where $a$ and $t$ are the limits of the operator, $\Gamma(\cdot)$ is the Euler's gamma function which is defined by the Euler integral of the second kind:

$$
\Gamma(\alpha)=\int_{0}^{\infty} \mathrm{e}^{-t} t^{\alpha-1} \mathrm{~d} t, \quad \alpha \in \mathbb{C}, \quad \operatorname{Re} \alpha>0 .
$$

Gamma function is a generalization of the factorial for noninteger arguments. The reduction formula holds:

$$
\Gamma(\alpha+1)=\alpha \Gamma(\alpha), \quad \alpha \in \mathbb{C}, \quad \operatorname{Re} \alpha>0 .
$$

For a special case $\alpha \in[0,1[$, the Riemann-Liouville fractional derivative is given by:

$$
{ }^{\mathrm{RL}} \mathrm{D}_{a, t}^{\alpha} f(t)=\frac{1}{\Gamma(n-\alpha)} \frac{\mathrm{d}}{\mathrm{d} t} \int_{a}^{t} \frac{f(s)}{(t-s)^{\alpha}} \mathrm{d} s, \quad t>\alpha .
$$

Definition 2. Let $f(\cdot) \in \mathcal{C}[a, b]$. The RiemannLiouville fractional integral of the order $\alpha \in \mathbb{C}, \operatorname{Re} \alpha>0$, is defined as [25]:

$$
\begin{aligned}
{ }^{\mathrm{RL}} \mathrm{I}_{a, t}^{\alpha} f(t) & \equiv{ }^{\mathrm{RL}} \mathrm{D}_{a, t}^{-\alpha} f(t) \\
& =\frac{1}{\Gamma(\alpha)} \int_{a}^{t}(t-s)^{\alpha-1} f(s) \mathrm{d} s, \quad t \in[a, b] .
\end{aligned}
$$

Definition 3. The Grünwald-Letnikov fractional derivative of $\alpha$-th order $\left(\alpha \in \mathbb{R}^{+}\right)$and fractional integral of $|\alpha|$-th order $\left(\alpha \in \mathbb{R}^{-}\right)$are given by [26]:

$$
{ }^{\mathrm{GL}} \mathrm{D}_{a, t}^{\alpha} f(t)=\lim _{h \rightarrow 0} \frac{1}{h^{\alpha}} \sum_{i=0}^{\left[\frac{t-a}{h}\right]}(-1)^{i}\left(\begin{array}{c}
\alpha \\
i
\end{array}\right) f(t-i h),
$$

where $[\cdot]$ is a floor operator, and $(\cdot)$ presents a generalized binomial coefficient defined by:

$$
\left(\begin{array}{c}
\alpha \\
i
\end{array}\right)=\frac{\Gamma(i-\alpha)}{\Gamma(-\alpha) \Gamma(i+1)}, \quad \alpha \in \mathbb{R}, \quad i \in \mathbb{N}_{0} .
$$

Definition 4. Let $f(\cdot)$ belong to the set of all $n$-th order differentiable functions on the finite interval $[a, b]$ :

$$
f(\cdot) \in \mathcal{C}^{n}[a, b]=\left\{f(t): \frac{\mathrm{d}}{\mathrm{d} t} f(t) \in \mathcal{C}^{n-1}[a, b]\right\},
$$

The Caputo fractional derivative of the order $\alpha$, for $\alpha \in \mathbb{C}, \quad \alpha \notin \mathbb{N}_{0}, \quad \operatorname{Re} \alpha \geq 0, \quad n-1 \leq \operatorname{Re} \alpha<n, \quad n \in \mathbb{N}, \quad$ is defined as [26]:

$$
\begin{gathered}
{ }^{\mathrm{C}} \mathrm{D}_{a, t}^{\alpha} f(t)=\frac{1}{\Gamma(n-\alpha)} \int_{a}^{t} \frac{f^{(n)}(s)}{(t-s)^{\alpha-n+1}} \mathrm{~d} s, \quad t \in[a, b], \\
f^{(n)}(s)=\left(\frac{\mathrm{d}}{\mathrm{d} s}\right)^{n} f(s),
\end{gathered}
$$

and for $\alpha \in \mathbb{N}_{0}$, it is given by [26]:

$$
{ }^{\mathrm{C}} \mathrm{D}_{a, t}^{\alpha} f(t)=\left(\frac{\mathrm{d}}{\mathrm{d} t}\right)^{\alpha} f(t), \quad t \in[a, b]
$$

\section{Previous Results Related to the Fractional-Order Time-Delay Systems}

A continuous time-invariant linear homogeneous fractional system including time-varying delays in state can be presented by a linear homogeneous fractional differential equation in a state space: 


$$
\begin{gathered}
{ }^{\mathrm{C}} \mathrm{D}_{t_{0}, t}^{\alpha} \mathbf{x}(t)=A_{0} \mathbf{x}(t)+\sum_{i=1}^{n} A_{i} \mathbf{x}\left(t-\tau_{x, i}(t)\right), \quad t \geq t_{0}, \\
\alpha \in] 0,1[,
\end{gathered}
$$

with the associated function of the initial state:

$$
\mathbf{x}(t)=\boldsymbol{\psi}_{x}(t), \quad t \in\left[t_{0}-\tau_{x, M}, t_{0}\right],
$$

where $\tau_{x, i}(t), \quad i=1,2, \ldots, n$, are time-varying delays in state that satisfy:

$$
\begin{gathered}
0<\tau_{x, i}(t) \leq \tau_{x, M}, \quad \forall i \in\{1,2, \ldots, n\}, \quad \forall t \in J, \\
J=\left[t_{0}, t_{0}+T[.\right.
\end{gathered}
$$

A linear nonhomogeneous fractional system including time-varying delays in state and input (control) can be described by a linear nonhomogeneous fractional statespace equation:

$$
\begin{aligned}
{ }^{\mathrm{C}} \mathrm{D}_{t_{0}, t}^{\alpha} \mathbf{x}(t)= & A_{0} \mathbf{x}(t)+\sum_{i=1}^{n} A_{i} \mathbf{x}\left(t-\tau_{x, i}(t)\right) \\
& +B_{0} \mathbf{u}(t)+\sum_{j=1}^{m} B_{j} \mathbf{u}\left(t-\tau_{u, j}(t)\right), \\
t \geq & \left.t_{0}, \quad \alpha \in\right] 0,1[
\end{aligned}
$$

with the associated function of the initial state:

$$
\mathbf{x}(t)=\boldsymbol{\Psi}_{x}(t), \quad t \in\left[t_{0}-\tau_{x, M}, t_{0}\right],
$$

and the associated function of the initial control:

$$
\mathbf{x}(t)=\boldsymbol{\psi}_{u}(t), \quad t \in\left[t_{0}-\tau_{u, M}, t_{0}\right],
$$

where $\tau_{x, i}(t), \quad i=1,2, \ldots, n$, and $\tau_{u, j}(t), \quad j=1,2, \ldots, m$, are time-varying delays in state and control, respectively, which satisfy:

$$
\begin{array}{cc}
0<\tau_{x, i}(t) \leq \tau_{x, M}, \quad \forall i \in\{1,2, \ldots, n\}, & \forall t \in J, \\
0<\tau_{u, j}(t) \leq \tau_{u, M}, \quad \forall j \in\{1,2, \ldots, m\}, & \forall t \in J, \\
J=\left[t_{0}, t_{0}+T[.\right. &
\end{array}
$$

A nonlinear nonhomogeneous perturbed fractional system including time-varying delays in state and control can be given by a nonlinear nonhomogeneous fractional state equation:

$$
\begin{aligned}
{ }^{\mathrm{C}} \mathrm{D}_{t_{0}, t}^{\alpha} \mathbf{x}(t)= & \left(A_{0}+\Delta A_{0}\right) \mathbf{x}(t) \\
& +\sum_{i=1}^{n}\left(A_{i}+\Delta A_{i}\right) \mathbf{x}\left(t-\tau_{x, i}(t)\right) \\
& +B_{0} \mathbf{u}(t)+\sum_{j=1}^{m} B_{j} \mathbf{u}\left(t-\tau_{u, j}(t)\right) \\
& +\mathbf{f}_{0}(\mathbf{x}(t))+\sum_{i=1}^{n} \mathbf{f}_{i}\left(\mathbf{x}\left(t-\tau_{x, i}(t)\right)\right), \\
t \geq & \left.t_{0}, \quad \alpha \in\right] 0,1[,
\end{aligned}
$$

with the associated functions of the initial state (15) and initial control (16), and with the time-varying delays satisfying (17). In the equations, $\mathbf{x}(\cdot) \in \mathbb{R}^{n}$ is the state vector, $\mathbf{u}(\cdot) \in \mathbb{R}^{m}$ is the given continuous vector function of input (control), $A_{i} \in \mathbb{R}^{n \times n}, \quad i=0,1,2, \ldots, n$, are the system matrices, the matrices $\Delta A_{i} \in \mathbb{R}^{n \times n}, i=0,1,2, \ldots, n$, present parameter perturbations of the system, $B_{j} \in \mathbb{R}^{n \times m}$, $j=0,1,2, \ldots, m$, are the input (control) matrices, $t_{0} \in \mathbb{R}$ is the initial time of observation of the system behavior, and $T$ is a positive number. Vector functions $\mathbf{f}_{0}(\cdot) \in \mathbb{R}^{n}$ and $\mathbf{f}_{i}(\cdot) \in \mathbb{R}^{n}, \quad i=1,2, \ldots, n, \quad$ present the nonlinear perturbations in respect to $\mathbf{x}(t)$ and $\mathbf{x}\left(t-\tau_{x, i}(t)\right)$, $i=1,2, \ldots, n$, respectively. It is assumed that:

$$
\begin{gathered}
\left\|\mathbf{f}_{0}(\mathbf{x}(t))\right\| \leq c_{0}\|\mathbf{x}(t)\|, \quad \forall t \geq t_{0}, \\
\left\|\mathbf{f}_{i}\left(\mathbf{x}\left(t-\tau_{x, i}(t)\right)\right)\right\| \leq c_{i}\left\|\mathbf{x}\left(t-\tau_{x, i}(t)\right)\right\|, \\
\forall i \in\{1,2, \ldots, n\}, \quad \forall t \geq t_{0},
\end{gathered}
$$

where $c_{i} \in \mathbb{R}^{+}, \forall i \in\{0,1,2, \ldots, n\}$, are known real positive constants. In this paper, the norm $\|(\cdot)\|$ denotes the Euclidean vector norm $\|\mathbf{x}(t)\|=\|\mathbf{x}(t)\|_{2}=\left(\mathbf{x}^{\mathrm{T}}(t) \mathbf{x}(t)\right)^{1 / 2}$ or the Euclidean matrix norm $\|A\|=\|A\|_{2}=\lambda_{\max }^{1 / 2}\left(A^{\mathrm{T}} A\right)=$ $\sigma_{\max }(A)$ induced by the Euclidean vector norm, where $\lambda_{\text {max }}(\cdot)$ and $\sigma_{\max }(\cdot)$ are the largest eigenvalue and the largest singular value of matrix $(\cdot)$, respectively.

The dynamic system behavior is observed over the time interval $J=\left[t_{0}, t_{0}+T[\right.$, where the quantity $T$ may be either a real positive number or the symbol $\infty$, so finitetime stability and practical stability may be considered simultaneously. System trajectories and control actions are bounded by the time-invariant sets that are defined a priori in a given problem. These sets are: $\mathcal{S}_{\delta}-$ the set of all initial states of the system, $\mathcal{S}_{\varepsilon}$ - the set of all allowable states of the system, $\mathcal{S}_{\alpha_{0}}$ - the set of all initial control actions, $\mathcal{S}_{\alpha_{u}}$ - the set of all allowable control actions, $\delta, \varepsilon, \alpha_{0}, \alpha_{u} \in \mathbb{R}^{+}, \quad \delta<\varepsilon$. These sets are assumed to be bounded, connected, and open. In this paper, the set $\mathcal{S}_{\rho}$ is defined as $\mathcal{S}_{\rho}=\{\mathbf{x}(t):\|\mathbf{x}(t)\|<\rho\}, \quad \rho \in \mathbb{R}^{+},[8-12]$.

The initial functions (15) and (16) and their norms can be given in general form as:

$$
\begin{gathered}
\boldsymbol{\Psi}_{x}(\cdot) \in \mathcal{C}\left(\left[t_{0}-\tau_{x, M}, t_{0}\right], \mathbb{R}^{n}\right), \\
\left\|\boldsymbol{\Psi}_{x}\right\|_{\mathcal{C}}=\underset{t \in\left[t_{0}-\tau_{x, M}, t_{0}\right]}{\max }\left\|\boldsymbol{\Psi}_{x}(t)\right\|, \\
\boldsymbol{\Psi}_{u}(\cdot) \in \mathcal{C}\left(\left[t_{0}-\tau_{u, M}, t_{0}\right], \mathbb{R}^{n}\right), \\
\left\|\boldsymbol{\Psi}_{u}\right\|_{\mathcal{C}}=\max _{t \in\left[t_{0}-\tau_{u, M}, t_{0}\right]}\left\|\boldsymbol{\Psi}_{u}(t)\right\|,
\end{gathered}
$$


where $\mathcal{C}\left(\left[t_{0}-\tau_{x, M}, t_{0}\right], \mathbb{R}^{n}\right)$ and $\mathcal{C}\left(\left[t_{0}-\tau_{u, M}, t_{0}\right], \mathbb{R}^{n}\right)$ denote the Banach spaces of all continuous real vector functions on time intervals $\left[t_{0}-\tau_{x, M}, t_{0}\right]$ and $\left[t_{0}-\tau_{u, M}, t_{0}\right]$, respectively, mapping these intervals into $\mathbb{R}^{n}$ with the topology of the uniform convergence. Here, it is assumed that the smoothness condition is present so that there is no difficulty with the questions of existence, uniqueness, and continuity of solutions of systems with respect to the initial conditions.

The definitions of the finite-time stability will be given for homogeneous system (11) and for nonhomogeneous system (14) or (18) with the associated initial functions.

Definition 5. The fractional delayed system given by a linear homogeneous state equation (11) satisfying the initial condition (12) is a finite-time stable with respect to $\left\{\delta, \varepsilon, t_{0}, J\right\}, \delta<\varepsilon$, if and only if:

$$
\left\|\Psi_{x}\right\|_{\mathcal{C}}<\delta
$$

implies:

$$
\|\mathbf{x}(t)\|<\varepsilon, \quad \forall t \in J .
$$

Definition 6. The fractional delayed system given by a nonhomogeneous linear (14) or nonlinear (18) state equation satisfying initial conditions (15) and (16) is a finite-time stable with respect to $\left\{\delta, \varepsilon, \alpha_{0}, \alpha_{u}, t_{0}, J\right\}$, $\delta<\varepsilon$, if and only if:

$$
\left\|\boldsymbol{\Psi}_{x}\right\|_{\mathcal{C}}<\delta, \quad\left\|\boldsymbol{\psi}_{u}\right\|_{\mathcal{C}}<\alpha_{0}
$$

and

$$
\|\mathbf{u}(t)\|<\alpha_{u}, \quad \forall t \in J
$$

imply:

$$
\|\mathbf{x}(t)\|<\varepsilon, \quad \forall t \in J .
$$

The finite-time stability analysis of nonlinear nonhomogeneous perturbed fractional systems with a constant time delay is suggested in [32]. The non-Lyapunov (finite-time) stability and stabilization of nonlinear nonhomogeneous perturbed fractional systems with timevarying delay is proposed in [33] for the system:

$$
\begin{aligned}
{ }^{\mathrm{C}} \mathrm{D}_{t_{0}, t}^{\alpha} \mathbf{x}(t)= & \left(A_{0}+\Delta A_{0}\right) \mathbf{x}(t) \\
& +\left(A_{1}+\Delta A_{1}\right) \mathbf{x}\left(t-\tau_{x}(t)\right) \\
& +B_{0} \mathbf{u}(t)+\mathbf{f}_{0}\left(\mathbf{x}(t), \mathbf{x}\left(t-\tau_{x}(t)\right)\right), \\
t \geq & \left.t_{0}, \quad \alpha \in\right] 0,1[
\end{aligned}
$$

with the initial function (12) and vector function $\mathbf{f}_{0}(\cdot)$ satisfying the assumption:

$$
\begin{aligned}
& \left\|\mathbf{f}_{0}\left(\mathbf{x}(t), \mathbf{x}\left(t-\tau_{x}(t)\right)\right)\right\| \\
& \quad \leq c_{0}\|\mathbf{x}(t)\|+c_{1}\left\|\mathbf{x}\left(t-\tau_{x}(t)\right)\right\|, \quad \forall t \geq t_{0},
\end{aligned}
$$

where $c_{0}, c_{1} \in \mathbb{R}^{+}$are known real positive constants.

Theorem 1. [33] The nonlinear nonhomogeneous fractional delayed system (26), satisfying the initial condition (12) and assumption (27), is a finite-time stable with respect to $\left\{\delta, \varepsilon, \alpha_{u}, t_{0}, J\right\}, \delta<\varepsilon$, if the following condition is satisfied:

$$
\begin{aligned}
\left(1+\frac{\mu_{\Sigma}\left(t-t_{0}\right)^{\alpha}}{\Gamma(\alpha+1)}\right) \mathrm{E}_{\alpha}\left(\mu_{\Sigma}\left(t-t_{0}\right)^{\alpha}\right) & \\
+ & \frac{\gamma_{u 0}\left(t-t_{0}\right)^{\alpha}}{\Gamma(\alpha+1)} \leq \frac{\varepsilon}{\delta}, \quad \forall t \in J,
\end{aligned}
$$

where:

$$
\begin{gathered}
\mu_{\Sigma}=\mu_{A_{0} c_{0}}+\mu_{A_{1} c_{1}}, \\
\mu_{A_{i} c_{i}}=\sigma_{\max }\left(A_{i}\right)+\sigma_{\max }\left(\Delta A_{i}\right)+c_{i}, \quad i=0,1, \\
\gamma_{u 0}=\frac{\alpha_{u}}{\delta} b_{0}, \quad b_{0}=\left\|B_{0}\right\|=\sigma_{\max }\left(B_{0}\right),
\end{gathered}
$$

and $\mathrm{E}_{\alpha}(\cdot)$ is the Mittag-Leffler function defined by:

$$
\mathrm{E}_{\alpha}(z)=\sum_{k=0}^{\infty} \frac{z^{k}}{\Gamma(k \alpha+1)}, \quad \alpha>0, \quad z \in \mathbb{C} .
$$

The finite-time stability of nonlinear nonhomogeneous fractional systems including multiple constant time delays in state is presented in [34] for the state equation:

$$
\begin{gathered}
{ }^{\mathrm{C}} \mathrm{D}_{t_{0}, t}^{\alpha} \mathbf{x}(t)=A_{0} \mathbf{x}(t)+\sum_{i=1}^{n} A_{i} \mathbf{x}\left(t-\tau_{x, i}\right)+B_{0} \mathbf{u}(t) \\
+\mathbf{f}_{0}(\mathbf{x}(t))+\sum_{i=1}^{n} \mathbf{f}_{i}\left(\mathbf{x}\left(t-\tau_{x, i}\right)\right), \\
\left.t \geq t_{0}, \quad \alpha \in\right] 0,1\left[, \quad 0<\tau_{x, 1}<\tau_{x, 2}<\cdots<\tau_{x, n}=\Delta,\right.
\end{gathered}
$$

with the associated function of the initial state:

$$
\mathbf{x}(t)=\boldsymbol{\psi}_{x}(t), \quad t \in\left[t_{0}-\Delta, t_{0}\right]
$$

and vector functions $\mathbf{f}_{i}(\cdot), i=0,1,2, \ldots, n$, satisfying the assumptions (19).

Theorem 2. [34] The nonlinear nonhomogeneous fractional delayed system (31), satisfying the initial condition (32) and assumptions (19), is a finite-time stable with respect to $\left\{\delta, \varepsilon, \alpha_{u}, t_{0}, J\right\}, \delta<\varepsilon$, if the following condition is satisfied:

$$
\left(1+\frac{\mu_{\Sigma}\left(t-t_{0}\right)^{\alpha}}{\Gamma(\alpha+1)}\right) \mathrm{e}^{\frac{\mu_{\Sigma}\left(t-t_{0}\right)^{\alpha}}{\Gamma(\alpha+1)}}+\frac{\gamma_{u 0}\left(t-t_{0}\right)^{\alpha}}{\Gamma(\alpha+1)} \leq \frac{\varepsilon}{\delta}
$$

$\forall t \in J$,

where:

$$
\begin{gathered}
\mu_{\Sigma}=\sum_{i=0}^{n} \mu_{A_{i} c_{i}}, \\
\mu_{A_{i} c_{i}}=\sigma_{\max }\left(A_{i}\right)+\sigma_{\max }\left(\Delta A_{i}\right)+c_{i}, \\
i=0,1,2, \ldots, n, \\
\gamma_{u 0}=\frac{\alpha_{u}}{\delta} b_{0}, \quad b_{0}=\left\|B_{0}\right\|=\sigma_{\max }\left(B_{0}\right) .
\end{gathered}
$$




\section{Main Results}

As a main contribution of this paper, the finite-time stability results are extended to the class of nonlinear nonhomogeneous perturbed fractional system with multiple time-varying delays in state and multiple time varying delays in control.

The sufficient conditions that enable system trajectories to stay within the a priori given sets for this class of systems are obtained. First, the conditions are obtained by using generalized Gronwall inequality, and then by using classical Bellman-Gronwall inequality.

Theorem 3. The nonlinear nonhomogeneous perturbed fractional delayed system (18), satisfying initial conditions (15) and (16) and assumptions (19), is a finite-time stable with respect to $\left\{\delta, \varepsilon, \alpha_{0}, \alpha_{u}, t_{0}, J\right\}, \delta<\varepsilon$, if the following condition is satisfied:

$$
\begin{gathered}
\left(1+\frac{\mu_{\Sigma}\left(t-t_{0}\right)^{\alpha}}{\Gamma(\alpha+1)}\right) \mathrm{E}_{\alpha}\left(\mu_{\Sigma}\left(t-t_{0}\right)^{\alpha}\right) \\
+\frac{\gamma_{u 0}\left(t-t_{0}\right)^{\alpha}}{\Gamma(\alpha+1)}+\frac{\gamma_{0 \Sigma} \tau_{u, M}^{\alpha}}{\Gamma(\alpha+1)} \\
+\frac{\gamma_{u \Sigma}\left(t-t_{0}-\tau_{u, M}\right)^{\alpha}}{\Gamma(\alpha+1)} \leq \frac{\varepsilon}{\delta}, \quad \forall t \in J,
\end{gathered}
$$

where:

$$
\begin{gathered}
\mu_{\Sigma}=\sum_{i=0}^{n} \mu_{A_{i} c_{i}}, \\
\mu_{A_{i} c_{i}}=\sigma_{\max }\left(A_{i}\right)+\sigma_{\max }\left(\Delta A_{i}\right)+c_{i}, \\
i=0,1,2, \ldots, n, \\
\gamma_{u 0}=\frac{\alpha_{u}}{\delta} b_{0}, \quad \gamma_{0 \Sigma}=\frac{\alpha_{0}}{\delta} \sum_{j=1}^{m} b_{j}, \quad \gamma_{u \Sigma}=\frac{\alpha_{u}}{\delta} \sum_{j=1}^{m} b_{j}, \\
b_{j}=\left\|B_{j}\right\|=\sigma_{\max }\left(B_{j}\right), \quad j=0,1,2, \ldots, m .
\end{gathered}
$$

Proof. In accordance with the property of the fractional order $\alpha \in] 0,1[$, one can obtain a solution in form of the equivalent Volterra integral equation:

$$
\begin{aligned}
& \mathbf{x}(t)=\mathbf{x}\left(t_{0}\right)+ \frac{1}{\Gamma(\alpha)} \times \\
& \times \int_{t_{0}}^{t}(t-s)^{\alpha-1}\left(\begin{array}{l}
\left(A_{0}+\Delta A_{0}\right) \mathbf{x}(s) \\
+\sum_{i=1}^{n}\left(A_{i}+\Delta A_{i}\right) \mathbf{x}\left(s-\tau_{x, i}(s)\right) \\
+B_{0} \mathbf{u}(s)+\sum_{j=1}^{m} B_{j} \mathbf{u}\left(s-\tau_{u, j}(s)\right) \\
+\mathbf{f}_{0}(\mathbf{x}(s))+\sum_{i=1}^{n} \mathbf{f}_{i}\left(\mathbf{x}\left(s-\tau_{x, i}(s)\right)\right)
\end{array}\right) \mathrm{d} s,
\end{aligned}
$$

Applying the norm on equation (37) and using the triangle inequality for vectors, an estimate of the solution $\mathbf{x}(t)$ is obtained:

$$
\begin{aligned}
& \|\mathbf{x}(t)\| \leq\left\|\mathbf{x}\left(t_{0}\right)\right\|+\frac{1}{\Gamma(\alpha)} \times \\
& \times \int_{t_{0}}^{t} \mid(t-s)^{\alpha-1 \mid}\left\|\begin{array}{l}
\sum_{i=1}^{n} \Delta A_{i} \mathbf{x}\left(s-\tau_{x, i}(s)\right)+B_{0} \mathbf{u}(s) \\
A_{0} \mathbf{x}(s)+\Delta A_{0} \mathbf{x}(s) \\
+\sum_{i=1}^{n} A_{i} \mathbf{x}\left(s-\tau_{x, i}(s)\right) \\
+\sum_{j=1}^{m} B_{j} \mathbf{u}\left(s-\tau_{u, j}(s)\right)+\mathbf{f}_{0}(\mathbf{x}(s)) \\
+\sum_{i=1}^{n} \mathbf{f}_{i}\left(\mathbf{x}\left(s-\tau_{x, i}(s)\right)\right)
\end{array}\right\|
\end{aligned}
$$

Now, applying the norm on equation (18) and taking into account the assumptions (19), it follows that:

$$
\begin{aligned}
\left\|{ }^{\mathrm{C}} \mathrm{D}_{t_{0}, t}^{\alpha} \mathbf{x}(t)\right\| \leq\left(\left\|A_{0}\right\|+\left\|\Delta A_{0}\right\|\right)\|\mathbf{x}(t)\| \\
\quad+\left(\left\|A_{1}\right\|+\left\|\Delta A_{1}\right\|\right)\left\|\mathbf{x}\left(t-\tau_{x, 1}(t)\right)\right\| \\
\quad+\cdots+\left(\left\|A_{n}\right\|+\left\|\Delta A_{n}\right\|\right)\left\|\mathbf{x}\left(t-\tau_{x, n}(t)\right)\right\|+\left\|B_{0}\right\|\|\mathbf{u}(t)\| \\
\quad+\sum_{j=1}^{m}\left\|B_{j}\right\|\left\|\mathbf{u}\left(t-\tau_{u, j}(t)\right)\right\|+c_{0}\|\mathbf{x}(t)\| \\
\quad+c_{1}\left\|\mathbf{x}\left(t-\tau_{x, 1}(t)\right)\right\|+\cdots+c_{n}\left\|\mathbf{x}\left(t-\tau_{x, n}(t)\right)\right\| .
\end{aligned}
$$

Taking into account $\left\|A_{i}\right\|=\sigma_{\max }\left(A_{i}\right)$, the inequality (39) can be written as:

$$
\begin{aligned}
& \left\|{ }^{\mathrm{C}} \mathrm{D}_{t_{0}, t}^{\alpha} \mathbf{x}(t)\right\| \leq\left(\sigma_{\max }\left(A_{0}\right)+\sigma_{\max }\left(\Delta A_{0}\right)+c_{0}\right)\|\mathbf{x}(t)\| \\
& +\left(\sigma_{\max }\left(A_{1}\right)+\sigma_{\max }\left(\Delta A_{1}\right)+c_{1}\right)\left\|\mathbf{x}\left(t-\tau_{x, 1}(t)\right)\right\| \\
& +\cdots+\left(\sigma_{\max }\left(A_{n}\right)+\sigma_{\max }\left(\Delta A_{n}\right)+c_{n}\right)\left\|\mathbf{x}\left(t-\tau_{x, n}(t)\right)\right\| \\
& +\left\|B_{0}\right\|\|\mathbf{u}(t)\|+\sum_{j=1}^{m}\left\|B_{j}\right\|\left\|\mathbf{u}\left(t-\tau_{u, j}(t)\right)\right\|,
\end{aligned}
$$

and using $\mu_{A_{i} c_{i}}=\sigma_{\max }\left(A_{i}\right)+\sigma_{\max }\left(\Delta A_{i}\right)+c_{i}, \quad i=0,1,2$, $\ldots, n$, and $b_{j}=\left\|B_{j}\right\|, \quad j=0,1,2, \ldots, m$ :

$$
\begin{aligned}
\left\|{ }^{\mathrm{C}} \mathrm{D}_{t_{0}, t}^{\alpha} \mathbf{x}(t)\right\| \leq & \mu_{A_{0} c_{0}}\|\mathbf{x}(t)\|+\mu_{A_{1} c_{1}}\left\|\mathbf{x}\left(t-\tau_{x, 1}(t)\right)\right\| \\
& +\cdots+\mu_{A_{n} c_{n}}\left\|\mathbf{x}\left(t-\tau_{x, n}(t)\right)\right\| \\
& +b_{0}\|\mathbf{u}(t)\|+\sum_{j=1}^{m} b_{j}\left\|\mathbf{u}\left(t-\tau_{u, j}(t)\right)\right\| .
\end{aligned}
$$

Using the inequality:

$$
\begin{gathered}
\left\|\mathbf{x}\left(t-\tau_{x, i}(t)\right)\right\| \leq \sup _{t^{*} \in\left[t-\tau_{x, M}, t\right]}\left\|\mathbf{x}\left(t^{\bullet}\right)\right\|, \\
\forall i \in\{1,2, \ldots, n\},
\end{gathered}
$$

the inequality (41) can be presented in the following manner: 
MIŠLJEN, M., LAZAREVIĆ, M.: NON-LYAPUNOV STABILITY OF THE FRACTIONAL-ORDER TIME-VARYING DELAY SYSTEMS

13

$$
\begin{aligned}
\left\|{ }^{\mathrm{C}} \mathrm{D}_{t_{0}, t}^{\alpha} \mathbf{x}(t)\right\| \leq & \mu_{\Sigma} \sup _{t^{\bullet} \in\left[t-\tau_{x, M}, t\right]}\left\|\mathbf{x}\left(t^{\bullet}\right)\right\|+b_{0}\|\mathbf{u}(t)\| \\
& +\sum_{j=1}^{m} b_{j}\left\|\mathbf{u}\left(t-\tau_{u, j}(t)\right)\right\|, \\
t> & t_{0}+\tau_{x, M},
\end{aligned}
$$

and then:

$$
\begin{aligned}
&\left\|\mathrm{C}^{\mathrm{C}} \mathrm{D}_{t_{0}, t}^{\alpha} \mathbf{x}(t)\right\| \leq \mu_{\Sigma}\left(\sup _{t \in\left[t-\tau_{x, M}, t\right]}\left\|\mathbf{x}\left(t^{\cdot}\right)\right\|+\left\|\boldsymbol{\Psi}_{x}\right\|_{\mathcal{C}}\right) \\
&+b_{0}\|\mathbf{u}(t)\|+\sum_{j=1}^{m} b_{j}\left\|\mathbf{u}\left(t-\tau_{u, j}(t)\right)\right\|, \\
& t>t_{0} .
\end{aligned}
$$

After combining (44) and (38), it follows:

$$
\begin{aligned}
& \|\mathbf{x}(t)\| \leq\left\|\boldsymbol{\Psi}_{x}\right\|_{\mathcal{C}}+\frac{1}{\Gamma(\alpha)} \times \\
& \quad \times \int_{t_{0}}^{t} \mid(t-s)^{\alpha-1 \mid} \| \begin{array}{l}
\mu_{\Sigma}\left(\sup _{t \in\left[t-\tau_{x, M}, t\right]}\left\|\mathbf{x}\left(t^{\cdot}\right)\right\|+\left\|\boldsymbol{\Psi}_{x}\right\|_{\mathcal{C}}\right) \\
\left.+b_{0}\|\mathbf{u}(t)\|+\sum_{j=1}^{m} b_{j}\left\|\mathbf{u}\left(t-\tau_{u, j}(t)\right)\right\|\right) \mathrm{d} s .
\end{array}
\end{aligned}
$$

Expanding (45) leads to:

$$
\begin{aligned}
\|\mathbf{x}(t)\| \leq & \left\|\boldsymbol{\Psi}_{x}\right\|_{\mathcal{C}}+\frac{1}{\Gamma(\alpha)} \int_{t_{0}}^{t}\left|(t-s)^{\alpha-1}\right| \mu_{\Sigma}\left\|\boldsymbol{\Psi}_{x}\right\|_{\mathcal{C}} \mathrm{d} s \\
& +\frac{1}{\Gamma(\alpha)} \int_{t_{0}}^{t}\left|(t-s)^{\alpha-1}\right| \mu_{\Sigma} \sup _{t^{*} \in\left[t-\tau_{x, M}, t\right]}\left\|\mathbf{x}\left(t^{\bullet}\right)\right\| \mathrm{d} s \\
& +\frac{1}{\Gamma(\alpha)} \int_{t_{0}}^{t}\left|(t-s)^{\alpha-1}\right| b_{0}\|\mathbf{u}(t)\| \mathrm{d} s \\
& +\frac{1}{\Gamma(\alpha)} \int_{t_{0}}^{t}\left|(t-s)^{\alpha-1}\right| \sum_{j=1}^{m} b_{j}\left\|\mathbf{u}\left(t-\tau_{u, j}(t)\right)\right\| \mathrm{d} s .
\end{aligned}
$$

Taking into account $\|\mathbf{u}(t)\|<\alpha_{u}$, and using $\tau_{u, M}$ instead of $\tau_{u, j}(t)$, it follows:

$$
\begin{aligned}
\|\mathbf{x}(t)\| \leq & \left\|\boldsymbol{\Psi}_{x}\right\|_{\mathcal{C}}+\frac{\mu_{\Sigma}\left\|\boldsymbol{\Psi}_{x}\right\|_{\mathcal{C}}}{\Gamma(\alpha)} \int_{t_{0}}^{t}\left|(t-s)^{\alpha-1}\right| \mathrm{d} s \\
& +\frac{\mu_{\Sigma}}{\Gamma(\alpha)} \int_{t_{0}}^{t}\left|(t-s)^{\alpha-1}\right|_{t^{\bullet} \in\left[t-\tau_{x, M}, t\right]} \sup _{\mid \mathbf{x}\left(t^{\bullet}\right) \| \mathrm{d} s} \\
& +\frac{b_{0} \alpha_{u}}{\Gamma(\alpha)} \int_{t_{0}}^{t}\left|(t-s)^{\alpha-1}\right| \mathrm{d} s \\
& +\frac{1}{\Gamma(\alpha)} \int_{t_{0}}^{t}\left|(t-s)^{\alpha-1}\right|\left\|\mathbf{u}\left(t-\tau_{u, M}\right)\right\| \mathrm{d} s \sum_{j=1}^{m} b_{j} .
\end{aligned}
$$

Integrating (47) leads to the following relations:

$$
\begin{aligned}
\|\mathbf{x}(t)\| \leq & \left\|\boldsymbol{\psi}_{x}\right\|_{\mathcal{C}}\left(1+\frac{\mu_{\Sigma}}{\Gamma(\alpha)} \frac{\left(t-t_{0}\right)^{\alpha}}{\alpha}\right) \\
& +\frac{\mu_{\Sigma}}{\Gamma(\alpha)} \int_{t_{0}}^{t}\left|(t-s)^{\alpha-1}\right|_{t^{\bullet} \in\left[\operatorname{Lup}_{x, M}, t\right]}\left\|\mathbf{x}\left(t^{\bullet}\right)\right\| \mathrm{d} s \\
& +\frac{\alpha_{u} b_{0}}{\Gamma(\alpha)} \frac{\left(t-t_{0}\right)^{\alpha}}{\alpha} \\
& +\frac{1}{\Gamma(\alpha)} \int_{t_{0}-\tau_{u, M}}^{t_{0}}\left|\left(t+\tau_{u, M}-s\right)^{\alpha-1}\right|\|\mathbf{u}(t)\| \mathrm{d} s \sum_{j=1}^{m} b_{j} \\
& +\frac{1}{\Gamma(\alpha)} \int_{t_{0}}^{t-\tau_{u, M}} \mid\left(t+\tau_{u, M}-s\right)^{\alpha-1}\|\mathbf{u}(t)\| \mathrm{d} s \sum_{j=1}^{m} b_{j},
\end{aligned}
$$

$$
\begin{aligned}
& \|\mathbf{x}(t)\| \leq\left\|\boldsymbol{\psi}_{x}\right\|_{\mathcal{C}}\left(1+\frac{\mu_{\Sigma}\left(t-t_{0}\right)^{\alpha}}{\Gamma(\alpha+1)}\right) \\
& +\frac{\mu_{\Sigma}}{\Gamma(\alpha)} \int_{t_{0}}^{t}\left|(t-s)^{\alpha-1}\right|_{t^{\bullet} \in\left[t-\tau_{x, M}, t\right]} \sup _{\mid} \mid \mathbf{x}\left(t^{\bullet}\right) \| \mathrm{d} s \\
& +\frac{\alpha_{u} b_{0}\left(t-t_{0}\right)^{\alpha}}{\Gamma(\alpha+1)} \\
& +\frac{1}{\Gamma(\alpha)} \int_{t_{0}-\tau_{u, M}}^{t_{0}}\left|(t-s)^{\alpha-1}\right| \mathrm{d} s\left\|\boldsymbol{\Psi}_{u}\right\|_{\mathcal{C}} \sum_{j=1}^{m} b_{j} \\
& +\frac{1}{\Gamma(\alpha)} \int_{t_{0}}^{t-\tau_{u, M}}\left|(t-s)^{\alpha-1}\right| \mathrm{d} s \alpha_{u} \sum_{j=1}^{m} b_{j}, \\
& \|\mathbf{x}(t)\| \leq\left\|\boldsymbol{\Psi}_{x}\right\|_{\mathcal{C}}\left(1+\frac{\mu_{\Sigma}\left(t-t_{0}\right)^{\alpha}}{\Gamma(\alpha+1)}\right) \\
& +\frac{\mu_{\Sigma}}{\Gamma(\alpha)} \int_{t_{0}}^{t}\left|(t-s)^{\alpha-1}\right| \sup _{t^{\bullet} \in\left[t-\tau_{x, M}, t\right]}\left\|\mathbf{x}\left(t^{\bullet}\right)\right\| \mathrm{d} s \\
& +\frac{\alpha_{u} b_{0}\left(t-t_{0}\right)^{\alpha}}{\Gamma(\alpha+1)}+\frac{1}{\Gamma(\alpha)} \frac{\tau_{u, M}^{\alpha}}{\alpha} \alpha_{0} \sum_{j=1}^{m} b_{j} \\
& +\frac{1}{\Gamma(\alpha)} \frac{\left(t-\tau_{u, M}-t_{0}\right)^{\alpha}}{\alpha} \alpha_{u} \sum_{j=1}^{m} b_{j}, \\
& \|\mathbf{x}(t)\| \leq\left\|\boldsymbol{\psi}_{x}\right\|_{\mathcal{C}}\left(1+\frac{\mu_{\Sigma}\left(t-t_{0}\right)^{\alpha}}{\Gamma(\alpha+1)}\right) \\
& +\frac{\mu_{\Sigma}}{\Gamma(\alpha)} \int_{t_{0}}^{t}\left|(t-s)^{\alpha-1}\right|_{t^{*} \in\left[t-\tau_{x, M}, t\right]}\left\|\mathbf{x}\left(t^{\cdot}\right)\right\| \mathrm{d} s \\
& +\frac{\alpha_{u} b_{0}\left(t-t_{0}\right)^{\alpha}}{\Gamma(\alpha+1)}+\frac{\alpha_{0} \tau_{u, M}^{\alpha}}{\Gamma(\alpha+1)} \sum_{j=1}^{m} b_{j} \\
& +\frac{\alpha_{u}\left(t-t_{0}-\tau_{u, M}\right)^{\alpha}}{\Gamma(\alpha+1)} \sum_{j=1}^{m} b_{j} .
\end{aligned}
$$


Introducing a nondecreasing function $g(t)$ in the following manner:

$$
g(t)=\left\|\boldsymbol{\psi}_{x}\right\|_{\mathcal{C}}\left(1+\frac{\mu_{\Sigma}\left(t-t_{0}\right)^{\alpha}}{\Gamma(\alpha+1)}\right)
$$

and using generalized Gronwall inequality [20], leads to:

$$
\|\mathbf{x}(t)\| \leq \sup _{t^{*} \in\left[t-\tau_{x, M}, t\right]}\left\|\mathbf{x}\left(t^{\cdot}\right)\right\| \leq g(t) \mathrm{E}_{\alpha}\left(\mu_{\Sigma}\left(t-t_{0}\right)^{\alpha}\right),
$$

and then to:

$$
\begin{aligned}
\|\mathbf{x}(t)\| \leq & \delta\left(1+\frac{\mu_{\Sigma}\left(t-t_{0}\right)^{\alpha}}{\Gamma(\alpha+1)}\right) \mathrm{E}_{\alpha}\left(\mu_{\Sigma}\left(t-t_{0}\right)^{\alpha}\right) \\
& +\frac{\alpha_{u} b_{0}\left(t-t_{0}\right)^{\alpha}}{\Gamma(\alpha+1)}+\frac{\alpha_{0} \tau_{u, M}^{\alpha}}{\Gamma(\alpha+1)} \sum_{j=1}^{m} b_{j} \\
& +\frac{\alpha_{u}\left(t-t_{0}-\tau_{u, M}\right)^{\alpha}}{\Gamma(\alpha+1)} \sum_{j=1}^{m} b_{j} .
\end{aligned}
$$

Finally, if the condition of Theorem 3 given by a relation (35) is used, it follows that:

$$
\|\mathbf{x}(t)\|<\varepsilon, \quad \forall t \in J .
$$

Based on the previous result, the following special cases can be obtained.

Theorem 4. The linear nonhomogeneous fractional delayed system (14) that satisfies the initial conditions (15) and (16) is a finite-time stable with respect to $\left\{\delta, \varepsilon, \alpha_{0}, \alpha_{u}, J_{0}\right\}, \delta<\varepsilon$, if the following condition holds:

$$
\begin{aligned}
&\left(1+\frac{\sigma_{\Sigma \max } t^{\alpha}}{\Gamma(\alpha+1)}\right) \mathrm{E}_{\alpha}\left(\sigma_{\Sigma \max } t^{\alpha}\right)+\frac{\gamma_{u 0} t^{\alpha}}{\Gamma(\alpha+1)} \\
&+\frac{\gamma_{0 \Sigma} \tau_{u, M}^{\alpha}}{\Gamma(\alpha+1)}+\frac{\gamma_{u \Sigma}\left(t-\tau_{u, M}\right)^{\alpha}}{\Gamma(\alpha+1)} \leq \frac{\varepsilon}{\delta}, \\
& \forall t \in J_{0}=[0, T[
\end{aligned}
$$

where:

$$
\sigma_{\Sigma \max }=\sum_{i=0}^{n} \sigma_{\max }\left(A_{i}\right)
$$

Theorem 5. The linear nonhomogeneous fractional delayed $\operatorname{system}(14)$, where $\mathbf{u}\left(t-\tau_{u, j}(t)\right) \equiv \mathbf{0}, \quad \forall j \in$ $\{1,2, \ldots, m\}$, satisfying the initial condition (15), is a finitetime stable with respect to $\left\{\delta, \varepsilon, \alpha_{u}, J_{0}\right\}, \delta<\varepsilon$, if the following condition is satisfied:

$$
\begin{gathered}
\left(1+\frac{\sigma_{\Sigma \max } t^{\alpha}}{\Gamma(\alpha+1)}\right) \mathrm{E}_{\alpha}\left(\sigma_{\Sigma \max } t^{\alpha}\right)+\frac{\gamma_{u 0} t^{\alpha}}{\Gamma(\alpha+1)} \leq \frac{\varepsilon}{\delta}, \\
\forall t \in J_{0} .
\end{gathered}
$$

Remark 1. If there are no delays in input (control) in the system (18), $\mathbf{u}\left(t-\tau_{u, j}(t)\right) \equiv \mathbf{0}, \forall j \in\{1,2, \ldots, m\}$, and there is a single delay in the state, then conditions given by Theorem 1 [33] can be obtained.

Remark 2. If there are no delays in input (control) in the system (18), $\mathbf{u}\left(t-\tau_{u, j}(t)\right) \equiv \mathbf{0}, \forall j \in\{1,2, \ldots, m\}$, and there are no parameter perturbations of the system, $\Delta A_{i}=0$, $\forall i \in\{0,1,2, \ldots, n\}$, and all delays are constant, $\tau_{x, i}(t)=$ $\tau_{x, i}=$ const., $\quad \forall i \in\{1,2, \ldots, n\}, \quad \tau_{u, j}(t)=\tau_{u, j}=$ const. $\forall j \in\{1,2, \ldots, m\}$, then conditions given by Theorem 2 [34] can be obtained.

Similarly, by using classical Bellman-Gronwall inequality (Appendix B - Lemma B.3), the following result can be obtained.

Theorem 6. The nonlinear nonhomogeneous perturbed fractional delayed system (18), satisfying initial conditions (15) and (16) and assumptions (19), is a finite-time stable with respect to $\left\{\delta, \varepsilon, \alpha_{0}, \alpha_{u}, t_{0}, J\right\}, \delta<\varepsilon$, if the following condition is satisfied:

$$
\begin{gathered}
\left(1+\frac{\mu_{\Sigma}\left(t-t_{0}\right)^{\alpha}}{\Gamma(\alpha+1)}\right) \mathrm{e}^{\frac{\mu_{\Sigma}\left(t-t_{0}\right)^{\alpha}}{\Gamma(\alpha+1)}}+\frac{\gamma_{u 0}\left(t-t_{0}\right)^{\alpha}}{\Gamma(\alpha+1)} \\
+\frac{\gamma_{0 \Sigma} \tau_{u, M}^{\alpha}}{\Gamma(\alpha+1)}+\frac{\gamma_{u \Sigma}\left(t-t_{0}-\tau_{u, M}\right)^{\alpha}}{\Gamma(\alpha+1)} \leq \frac{\varepsilon}{\delta}, \\
\forall t \in J,
\end{gathered}
$$

where:

$$
\begin{gathered}
\mu_{\Sigma}=\sum_{i=0}^{n} \mu_{A_{i} c_{i}}, \\
\mu_{A_{i} c_{i}}=\sigma_{\max }\left(A_{i}\right)+\sigma_{\max }\left(\Delta A_{i}\right)+c_{i}, \\
i=0,1,2, \ldots, n, \\
\gamma_{u 0}=\frac{\alpha_{u}}{\delta} b_{0}, \quad \gamma_{0 \Sigma}=\frac{\alpha_{0}}{\delta} \sum_{j=1}^{m} b_{j}, \quad \gamma_{u \Sigma}=\frac{\alpha_{u}}{\delta} \sum_{j=1}^{m} b_{j}, \\
b_{j}=\left\|B_{j}\right\|=\sigma_{\max }\left(B_{j}\right), \quad j=0,1,2, \ldots, m .
\end{gathered}
$$

Proof. The proof immediately follows from the proof of Theorem 3 and applying the Bellman-Gronwall inequality (Lemma B.3). For the sake of brevity, the proof of Theorem 6 is omitted here.

Also, from Theorem 3, the finite-time stability condition for classical (integer-order) system can be obtained.

Theorem 7. The nonlinear nonhomogeneous perturbed integer-order $(\alpha=1)$ delayed system (18), satisfying the initial conditions (15) and (16) and assumptions (19), is a finite-time stable with respect to $\left\{\delta, \varepsilon, \alpha_{0}, \alpha_{u}, t_{0}, J\right\}$, $\delta<\varepsilon$, if the following condition is satisfied:

$$
\begin{gathered}
\left(1+\mu_{\Sigma}\left(t-t_{0}\right)\right) \mathrm{e}^{\mu_{\Sigma}\left(t-t_{0}\right)}+\gamma_{u 0}\left(t-t_{0}\right) \\
\quad+\gamma_{0 \Sigma} \tau_{u, M}+\gamma_{u \Sigma}\left(t-t_{0}-\tau_{u, M}\right) \leq \frac{\varepsilon}{\delta}, \\
\forall t \in J, \quad \Gamma(2)=1, \quad \mathrm{E}_{1}(\cdot)=\mathrm{e}^{(\cdot)} .
\end{gathered}
$$

Remark 3. If ( $\alpha=1)$ and there is a single constant delay in 
state and a single constant delay in control, and taking into account condition (61), one can obtain the same condition which is related to integer-order time-delay systems (see [35]).

The proposed results can be applied to any fractionalorder or integer-order time-delay model. An example of time-delay model can be found in [36, pp. 261-262]. Recently, the finite-time stability for a class of fractionalorder delayed neural networks as well as for the fractionalorder complex-valued memristor based neural networks including time-varying delays was considered and presented in $[37,38]$, respectively.

\section{Numerical Example}

A nonlinear nonhomogeneous perturbed fractional system with multiple time-varying delays in state and control is given by the state equation:

$$
\begin{aligned}
{ }^{\mathrm{C}} \mathrm{D}_{0, t}^{1 / 2} \mathbf{x}(t)= & \left(A_{0}+\Delta A_{0}\right) \mathbf{x}(t) \\
& +\left(A_{1}+\Delta A_{1}\right) \mathbf{x}\left(t-\tau_{x, 1}(t)\right) \\
& +\left(A_{2}+\Delta A_{2}\right) \mathbf{x}\left(t-\tau_{x, 2}(t)\right)+B_{0} \mathbf{u}(t) \\
& +B_{1} \mathbf{u}\left(t-\tau_{u, 1}(t)\right)+B_{2} \mathbf{u}\left(t-\tau_{u, 2}(t)\right) \\
& +\mathbf{f}_{0}(\mathbf{x}(t))+\mathbf{f}_{1}\left(\mathbf{x}\left(t-\tau_{x, 1}(t)\right)\right) \\
& +\mathbf{f}_{2}\left(\mathbf{x}\left(t-\tau_{x, 2}(t)\right)\right) \\
t \geq & 0
\end{aligned}
$$

where:

$$
\begin{gathered}
A_{0}=\left[\begin{array}{ll}
-0,2 & 0 \\
-0,1 & 0,3
\end{array}\right], \quad \Delta A_{0}=\left[\begin{array}{rr}
-0,02 & 0,01 \\
-0,01 & 0,03
\end{array}\right], \\
A_{1}=\left[\begin{array}{cc}
-0,2 & 0,1 \\
0 & -0,1
\end{array}\right], \quad \Delta A_{1}=\left[\begin{array}{rr}
-0,05 & 0,01 \\
0,02 & -0,03
\end{array}\right], \\
A_{2}=\left[\begin{array}{cc}
0,3 & 0 \\
-0,05 & 0,2
\end{array}\right], \quad \Delta A_{2}=\left[\begin{array}{ll}
0,04 & 0 \\
0 & 0,02
\end{array}\right], \\
B_{0}=\left[\begin{array}{ll}
0 & -3 \\
1 & 0
\end{array}\right], \quad B_{1}=\left[\begin{array}{ll}
2 & 0 \\
0 & 1
\end{array}\right], \quad B_{2}=\left[\begin{array}{ll}
0 & 0 \\
0 & -1
\end{array}\right], \\
t_{0}=0, \quad \tau_{x, M}=0,01 \mathrm{~s}, \quad \tau_{u, M}=0,03 \mathrm{~s}, \\
c_{0}=0,02, \quad c_{1}=0,05, \quad c_{2}=0,04 .
\end{gathered}
$$

Initial conditions are:

$$
\begin{aligned}
& \mathbf{x}(t)=\boldsymbol{\psi}_{x}(t)=\left[\begin{array}{ll}
0,04 & 0,05
\end{array}\right]^{\mathrm{T}}, \\
& t \in\left[t_{0}-\tau_{x, M}, t_{0}\right]=[-0,01 ; 0] \text {, } \\
& \mathbf{u}(t)=\boldsymbol{\Psi}_{u}(t)=\left[\begin{array}{ll}
0,02 & 0
\end{array}\right]^{\mathrm{T}}, \\
& t \in\left[t_{0}-\tau_{u, M}, t_{0}\right]=[-0,03 ; 0] \text {, }
\end{aligned}
$$

The task is to analyze the finite-time stability with respect to $\left\{\delta=0,1 ; \varepsilon=500 ; \alpha_{0}=0,2 ; \alpha_{u}=2 ; J_{0}=[0,3] \mathrm{s}\right\}$. From the initial conditions and given state equation, it follows:

$$
\begin{aligned}
& \left\|\boldsymbol{\Psi}_{x}\right\|_{\mathcal{C}}=\max _{t \in[-0,01 ; 0]}\left\|\boldsymbol{\Psi}_{x}(t)\right\|=\left\|\boldsymbol{\Psi}_{x}\right\| \\
& =\left(0,04^{2}+0,05^{2}\right)^{1 / 2}=0,064<\delta=0,1, \\
& \left\|\boldsymbol{\Psi}_{u}\right\|_{\mathcal{C}}=\max _{t \in[-0,03 ; 0]}\left\|\boldsymbol{\Psi}_{u}(t)\right\|=\left\|\boldsymbol{\Psi}_{u}\right\| \\
& =\left(0,02^{2}+0^{2}\right)^{1 / 2}=0,02<\alpha_{0}=0,2, \\
& \sigma_{\max }\left(A_{0}\right)=\lambda_{\max }^{1 / 2}\left(A_{0}^{\mathrm{T}} A_{0}\right)=0,3257, \\
& \sigma_{\max }\left(\Delta A_{0}\right)=\lambda_{\max }^{1 / 2}\left(\Delta A_{0}^{\mathrm{T}} \Delta A_{0}\right)=0,0362 \text {, } \\
& \sigma_{\max }\left(A_{1}\right)=\lambda_{\max }^{1 / 2}\left(A_{1}^{\mathrm{T}} A_{1}\right)=0,2288, \\
& \sigma_{\max }\left(\Delta A_{1}\right)=\lambda_{\max }^{1 / 2}\left(\Delta A_{1}^{\mathrm{T}} \Delta A_{1}\right)=0,04, \\
& \sigma_{\max }\left(A_{2}\right)=\lambda_{\max }^{1 / 2}\left(A_{2}^{\mathrm{T}} A_{2}\right)=0,3071, \\
& \sigma_{\max }\left(\Delta A_{2}\right)=\lambda_{\max }^{1 / 2}\left(\Delta A_{2}^{\mathrm{T}} \Delta A_{2}\right)=0,2146, \\
& \mu_{A_{0} c_{0}}=\sigma_{\max }\left(A_{0}\right)+\sigma_{\max }\left(\Delta A_{0}\right)+c_{0}=0,3819, \\
& \mu_{A_{1} c_{1}}=\sigma_{\max }\left(A_{1}\right)+\sigma_{\max }\left(\Delta A_{1}\right)+c_{1}=0,3188, \\
& \mu_{A_{2} c_{2}}=\sigma_{\max }\left(A_{2}\right)+\sigma_{\max }\left(\Delta A_{2}\right)+c_{2}=0,5617 \text {, } \\
& \mu_{\Sigma}=\mu_{A_{0} c_{0}}+\mu_{A_{1} c_{1}}+\mu_{A_{2} c_{2}}=1,2624
\end{aligned}
$$

$$
\begin{aligned}
& b_{0}=\left\|B_{0}\right\|=\sigma_{\max }\left(B_{0}\right)=3, \quad \gamma_{u 0}=\frac{\alpha_{u}}{\delta} b_{0}=60, \\
& b_{1}=\left\|B_{1}\right\|=\sigma_{\max }\left(B_{1}\right)=2, \quad \gamma_{0 \Sigma}=\frac{\alpha_{0}}{\delta}\left(b_{1}+b_{2}\right)=6, \\
& b_{2}=\left\|B_{2}\right\|=\sigma_{\max }\left(B_{2}\right)=1, \quad \gamma_{u \Sigma}=\frac{\alpha_{u}}{\delta}\left(b_{1}+b_{2}\right)=60 .
\end{aligned}
$$

Using the condition of Theorem 3, given by (35), it holds:

$$
\begin{aligned}
& \left(1+\frac{1,2624 T_{\mathrm{e}}^{1 / 2}}{\Gamma(3 / 2)}\right) \mathrm{E}_{1 / 2}\left(1,2624 T_{\mathrm{e}}^{1 / 2}\right) \\
& +\frac{60 T_{\mathrm{e}}^{1 / 2}}{\Gamma(3 / 2)}+\frac{6 \times 0,03^{1 / 2}}{\Gamma(3 / 2)}+\frac{60\left(T_{\mathrm{e}}-0,03\right)^{1 / 2}}{\Gamma(3 / 2)} \leq \frac{500}{0,1} .
\end{aligned}
$$

From (69), the estimated time of the finite-time stability is:

$$
T_{\mathrm{e}} \approx 4 \mathrm{~s} \text {. }
$$

The system (62) with the initial conditions (64) is a finitetime stable over the time interval $J_{0}=[0,3] \mathrm{s}$.

Theorem 6 can also be used to check the finite-time stability of a given system. Using the condition of Theorem 6 , given by (59), it holds:

$$
\begin{aligned}
& \left(1+\frac{1,2624 T_{\mathrm{e}}^{1 / 2}}{\Gamma(3 / 2)}\right) \mathrm{e}^{\frac{1,2624 T_{\mathrm{e}}^{1 / 2}}{\Gamma(3 / 2)}}+\frac{60 T_{\mathrm{e}}^{1 / 2}}{\Gamma(3 / 2)} \\
& +\frac{6 \times 0,03^{1 / 2}}{\Gamma(3 / 2)}+\frac{60\left(T_{\mathrm{e}}-0,03\right)^{1 / 2}}{\Gamma(3 / 2)} \leq \frac{500}{0,1} .
\end{aligned}
$$


From (71), the estimated time of the finite-time stability is:

$$
T_{\mathrm{e}} \approx 20,1 \mathrm{~s} \text {. }
$$

This result also shows that the system (62) with the initial conditions (64) is a finite-time stable over the time interval $J_{0}=[0,3] \mathrm{s}$.

All these conditions are only sufficient. If the obtained estimated time is equal or larger than the given time, it always means that the given system will be stable over the given time interval. On the other hand, if the obtained estimated time is smaller than the given time, it does not mean that the given system will not be stable over the given time interval. In the previous example, both results give estimated times that are larger than the given time.

\section{Conclusion}

This paper deals with the non-Lyapunov stability of the fractional-order time-delay systems. The main features of the finite-time and practical stability are extended to the class of nonlinear nonhomogeneous perturbed fractional systems including multiple time-varying delays in state and multiple time-varying delays in input (control). Sufficient conditions of stability are obtained for the given class of systems using generalized Gronwall inequality. The illustrative example is given to support the obtained analytical result.

\section{Appendix A - Notations}

$$
\begin{aligned}
& A \quad \text { - system matrix } \\
& B \text { - input (control) matrix } \\
& \mathrm{E}_{\alpha}(\cdot) \text { - Mittag-Leffler function } \\
& \mathbf{f}(\cdot) \text { - nonlinear perturbation } \\
& t \quad-\text { time } \\
& \mathbf{u}(\cdot) \quad \text { - input (control) vector } \\
& \mathbf{x}(\cdot) \quad \text { - state vector } \\
& \Gamma(\cdot) \text { - Euler's gamma function } \\
& \lambda(\cdot) \text { - eigenvalue of matrix }(\cdot) \\
& \Sigma \text { - summation } \\
& \sigma(\cdot) \quad \text { - singular value of matrix (.) } \\
& \tau_{u}(\cdot) \text { - time delay in input (control) } \\
& \tau_{x}(\cdot) \text { - time delay in state } \\
& \boldsymbol{\psi}_{u}(\cdot) \text { - function of initial input (control) } \\
& \boldsymbol{\psi}_{x}(\cdot) \text { - function of initial state } \\
& \mathbb{N} \quad \text { - set of all positive integers } \\
& \mathbb{N}_{0} \quad \text { - set of all nonnegative integers } \\
& \mathbb{R} \quad \text { - set of all real numbers } \\
& \mathbb{R}^{+} \quad \text { - set of all positive real numbers } \\
& \mathbb{R}^{-} \quad \text { - set of all negative real numbers } \\
& \mathbb{R}_{+} \quad \text { - set of all nonnegative real numbers } \\
& \mathbb{C} \quad \text { - set of all complex numbers } \\
& \mathbb{R}^{n} \quad \text { - } n \text {-dimensional real vector space } \\
& \mathbb{R}^{n \times m}-\text { set of all real } n \times m \text { matrices } \\
& \text { max - maximum } \\
& \text { sup - supremum }
\end{aligned}
$$

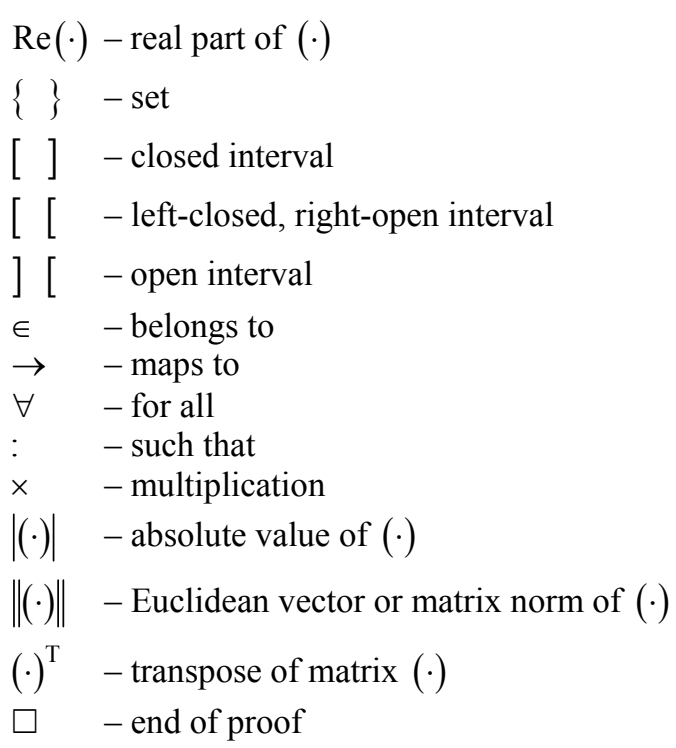

\section{Appendix B}

Lemma B.1. (Generalized Gronwall inequality [20]) Suppose $x(\cdot): \mathbb{R} \rightarrow \mathbb{R}$ and $a(\cdot): \mathbb{R} \rightarrow \mathbb{R}$ are nonnegative and integrable in every closed and bounded subinterval of $[0, T[$ and $g(\cdot):[0, T[\rightarrow \mathbb{R}$ is nonnegative, nondecreasing, continuous, and bounded function such that:

$$
x(t) \leq a(t)+g(t) \int_{0}^{t}(t-s)^{\alpha-1} x(s) \mathrm{d} s, \quad \alpha>0 .
$$

Then for $t \in[0, T[$ :

$$
x(t) \leq a(t)+\int_{0}^{t} \sum_{n=1}^{\infty} \frac{(g(t) \Gamma(\alpha))^{n}}{\Gamma(n \alpha)}(t-s)^{n \alpha-1} a(s) \mathrm{d} s .
$$

Lemma B.2. [20] Under the hypothesis of Lemma B.1, if $a(t)$ is nondecreasing, then:

$$
x(t) \leq a(t) \mathrm{E}_{\alpha}\left(g(t) \Gamma(\alpha) t^{\alpha}\right) .
$$

Lemma B.3. (the Bellman-Gronwall inequality) If $x(\cdot): \mathbb{R}_{+} \rightarrow \mathbb{R}_{+}$is bounded continuous function on each closed interval $[0, t]$ and satisfies:

$$
x(t) \leq a(t)+\int_{0}^{t} g(s) x(s) \mathrm{d} s
$$

for nondecreasing function $a(\cdot)$ and nonnegative integrable function $g(\cdot)$, then:

$$
x(t) \leq a(t) \exp \left(\int_{0}^{t} g(s) \mathrm{d} s\right)
$$

\section{Acknowledgement}

This research was sponsored by the research grants of the Ministry of Education, Science and Technological 
Development of the Republic of Serbia under the number TR 35006: "Sustainability and improvement of machine systems in the energetics and transport by application of forensic engineering, eco and robust design" (2011.-2015.).

\section{References}

[1] DELBOSCO, D., RODINO, L.: Existence and Uniqueness for a Nonlinear Fractional Differential Equation, Journal of Mathematical Analysis and Applications, 1996, Vol. 204, No. 2, pp. 609-625.

[2] ZHANG, X.: Some Results of Linear Fractional Order Time-Delay System, Applied Mathematics and Computation, 2008, Vol. 197, No. 1, pp. 407-411.

[3] RICHARD, J.-P.: Time Delay Systems: An Overview of some Recent Advances and Open Problems, Automatica, 2003, Vol. 39, No. 10, pp. 1667-1694.

[4] ZAVAREI, M., JAMSHIDI, M.: Time-Delay Systems: Analysis, Optimization and Applications, North-Holland, Amsterdam, 1987.

[5] DEBElJKOVIĆ, D., STOJANOVIĆ, S., NESTOROVIĆ, T., DIMITRIJEVIĆ, N.: Finite Time and Practical Stability of Linear Continuous Time Delay Systems - Classical and Modern Approach: An Overview, Scientific Technical Review, ISSN 1820-0206, 2013, Vol. 63, No. 1, pp. 25-35.

[6] LEE, T. N., DIANT, T. S.: Stability of Time Delay Systems, IEEE Transactions on Automatic Control, 1981, Vol. AC-31, No. 3, pp. 951-953.

[7] CHEN, J., XU, D., SHAFAI, B.: On Sufficient Conditions for Stability Independent of Delay, IEEE Transactions on Automatic Control, 1995, Vol. AC-40, No. 9, pp. 1675-1680.

[8] LAZAREVIĆ, M. P., DEBELJKOVIĆ, D. LJ., NENADIĆ, Z. LJ., MILINKOVIĆ, S. A.: Finite-Time Stability of Delayed Systems, IMA Journal of Mathematical Control and Information, 2000, Vol. 17, No. 2, pp. 101-109.

[9] DEBElJKOVIĆ, D. LJ., LAZAREViĆ, M. P., KORUGA, Đ., MILINKOVIĆ, S. A., JOVANOVIĆ, M. B., JACIĆ, LJ. A.: Further Results on Non-Lyapunov Stability of the Linear Nonautonomous Systems with Delayed State, Journal Facta Universitatis, Niš, Serbia, 2001, Vol. 3, No. 11, pp. 231-241.

[10] DEBELJKOVIĆ, D. LJ., LAZAREVIĆ, M. P., MILINKOVIĆ, S. A., JOVANOVIĆ, M. B.: Finite Time Stability Analysis of Linear Time Delay System: Bellman-Gronwall Approach, IFAC International Workshop Linear Time Delay Systems, Grenoble, France, 6-7 July, 1998, pp. 171-176.

[11] DEBELJKOVIĆ, D. LJ., BUZUROVIĆ, I. M., JOVANOVIĆ, A. M., SIMEUNOVIĆ, G. V., On Finite Time Stability of Continuous Time Delayed Systems: New Delay Dependent Conditions, The $27^{\text {th }}$ Chinese Control and Decision Conference (CCDC), 2015, pp. 53645369.

[12] GRUJIĆ, LJ. T.: Non-Lyapunov Stability Analysis of Large-Scale Systems on Time-Varying Sets, International Journal of Control, 1975, Vol. 21, No. 3, pp. 401-415.

[13] WEISS, L., INFANTE, E. F.: On the Stability of Systems Defined over a Finite Time Interval, Proceedings of the National Academy of Sciences, 1965, Vol. 54, No. 1, pp .44-48.

[14] LASHIRER, A. M., STORY, C.: Final-Stability with Some Applications, IMA Journal of Applied Mathematics, 1972, Vol. 9, No. 3, pp. 379-410.

[15] CHEN, Q., AHN, H., PODLUBNY, I.: Robust Stability Check of Fractional Order Linear Time Invariant Systems with Interval Uncertainties, Journal Signal Processing, 2006, Vol. 86, No. 10, pp. 2611-2618.

[16] MATIGNON, D.: Stability Properties for Generalized Fractional Differential Systems, ESAIM: Proceedings, December, 1998, Vol. 5, pp. 145-158.

[17] DENG, W., LI, C., LU, J., Stability Analysis of Linear Fractional Differential System with Multiple Time Delays, Nonlinear Dynamics, 2007, Vol. 48, No. 4, pp. 409-416.

[18] CHEN, Y. Q., MOORE, K. L.: Analytical Stability Bound for a Class of Delayed Fractional-Order Dynamic Systems, Nonlinear Dynamics, 2002, Vol. 29, No. 1, pp. 191-200.

[19] LAZAREVIĆ, M. P.: Finite Time Stability Analysis of $P D^{\alpha}$ Fractional Control of Robotic Time-Delay Systems, Mechanics Research Communications, 2006, Vol. 33, No. 2, pp. 269-279.
[20] YE, H., GAO, J., DING, Y.: A Generalized Gronwall Inequality and its Application to a Fractional Differential Equation, Journal of Mathematical Analysis and Applications, 2007, Vol. 328, No. 2, pp. 1075-1081.

[21] LAZAREVIĆ, M. P., SPASIĆ, A. M.: Finite-Time Stability Analysis of Fractional Order Time-Delay Systems: Gronwall's Approach, Mathematical and Computer Modelling, 2009, Vol. 49, No. 3-4, pp. 475-481.

[22] YANG X., SONG Q., LIU Y., ZHAO Z., Finite-Time Stability Analysis of Fractional-Order Neural Networks with Delay, Neurocomputing, 2015, Vol. 152, pp. 19-26.

[23] KE, Y., MIAO, C.: Stability Analysis of Fractional-Order CohenGrossberg Neural Networks with Time Delay, International Journal of Computer Mathematics, 2015, Vol. 92, No. 6, pp. 1102-1113.

[24] PETRAS, I., DORCAK, L., O'LEARY, P., VINAGRE, B. M., PODLUBNY, I.: The Modelling and Analysis of Fractional-Order Control Systems in Frequency Domain, Proceedings of the ICCC'2000 Conference, High Tatras, 23-26 May, 2000, pp. 261264.

[25] KILBAS, A. A., SRIVASTAVA, H. M., TRUJILlO, J. J.: Theory and Applications of Fractional Differential Equations, Elsevier, Amsterdam, 2006.

[26] PODLUBNY, I.: Fractional Differential Equations, Academic Press, San Diego, California, USA, 1999.

[27] OLDHAM, K. B., SPANIER, J.: The Fractional Calculus: Theory and Applications of Differentiation and Integration to Arbitrary Order, Academic Press, New York, NY, USA, 1974.

[28] HILFER, R.: Applications of Fractional Calculus in Physics, World Scientific, River Edge, NJ, USA, 2000.

[29] LAZAREVIĆ, M.: Further Results on Fractional Order Control of a Mechatronic System, Scientific Technical Review, ISSN 18200206, 2013, Vol. 63, No. 3, pp. 22-32.

[30] HSU, J. P, SPASIĆ, A.: Interfacial Electrovicoelasticity and Electrophoresis, CRC Press Taylor \& Francis Group, Boca Raton, FL 33487-2742, 2010.

[31] LAZAREVIĆ, M.: Some New Results on Iterative Learning Control of Noninteger Order, Scientific Technical Review, ISSN 1820-0206, 2014, Vol. 64, No. 2, pp. 33-41.

[32] LAZAREVIĆ, M., OBRADOVIĆ, A., VASIĆ, V.: Robust FiniteTime Stability Analysis of Fractional Order Time Delay Systems: New Results, Proceedings of the $6^{\text {th }}$ WSEAS International Conference on Dynamical Systems and Control, Control '10, Tunisia, May, 2010, pp. 101-106.

[33] LAZAREVIĆ, M. P.: Non-Lyapunov Stability and Stabilization of Fractional Order Systems Including Time-Varying Delays, Recent Researches in System Science, Proceedings of the $15^{\text {th }}$ WSEAS International Conference on Systems (Part of $15^{\text {th }}$ WSEAS CSCC Multiconference), Corfu, 14-16 July, 2011, pp. 196-201.

[34] LAZAREVIĆ, M.: Finite-Time Stability Analysis of Fractional Order Time Delay Systems: Bellman-Gronwall's Approach, Scientific Technical Review, ISSN 1820-0206, 2007, Vol. LVII, No. 1, pp. 8-15.

[35] DEBELJKOVIĆ, D. LJ., LAZAREVIĆ, M. P., KORUGA, DJ., MILINKOVIĆ, S. A., JOVANOVIĆ, M. B., JACIĆ, LJ. A.: Further Results on Non-Lyapunov Stability of the Linear Nonautonomous Systems with Delayed State, Journal Facta Universitatis, Niš, Serbia, Yugoslavia, 2001, Vol. 3, No. 11, pp. 231-241.

[36] DEBELJKOVIĆ, D. LJ., BUZUROVIĆ, I. M.: Dynamics of Singular and Descriptive Time Delayed Control Systems: Stability, Robustness, Optimization, Stabilizability and Robustness Stabilizability, University of Belgrade, Faculty of Mechanical Engineering, Belgrade, 2013.

[37] CHEN, L., LIU, C., WU, R., HE, Y., CHAI, Y.: Finite-Time Stability Criteria for a Class of Fractional-Order Neural Networks with Delay, Neural Computing and Applications, Springer London, 2015, pp. 1-8.

[38] RAKKIYAPPAN, R., VELMURUGAN, G., CAO, J.: Finite-Time Stability Analysis of Fractional-Order Complex-Valued MemristorBased Neural Networks with Time Delays, Nonlinear Dynamics, Springer Netherlands, 2014, Vol. 78, No. 4, pp. 2823-2836.

Received: 05.06.2015. Accepted: 12.08.2015. 


\title{
Neljapunovska stabilnost sistema necelog reda sa vremenski promenljivim kašnjenjima
}

\begin{abstract}
U ovom radu, kriterijumi stabilnosti na konačnom vremenskom intervalu su prošireni na nelinearne nehomogene perturbovane sisteme necelobrojnog reda koji sadrže višestruka vremenski promenljiva kašnjenja. Dobijeni su dovoljni uslovi stabilnosti za sisteme necelog reda sa višestrukim vremenskim kašnjenjima korišćenjem generalizovanog i klasičnog Gronwallovog pristupa. Numerički primer je dat u cilju ilustracije značaja dobijenog rezultata.
\end{abstract}

Ključne reči: kontinualni sistem, nelinearni sistem, vremensko kašnjenje, stabilnost sistema, neljapunovska stabilnost, stabilnost na konačnom vremenskom intervalu, sistem necelobrojnog reda.

\section{Stabilité de non Lyapunov de l'ordre fractionnel à délai temporel variable}

Dans ce papier les critères de stabilité sur l'intervalle temporelle finie sont élargis sur les systèmes non linéaires, non homogènes et perturbés de l'ordre fractionnel qui comportent multiples délais variables temporellement . On a obtenu les conditions suffisantes de la stabilité pour les systèmes de l'ordre fractionnel à multiple délai temporel par utilisation de l'approche classique et généralisée de Gronwall. L'exemple numérique a été donné dans le but d'illustrer l'importance du résultat obtenu.

Mots clés: système continu, système non linéaire, système à délai, délai temporel, stabilité de système, stabilité de non Lyapunov, stabilité sur intervalle temporelle finie, système de l'ordre fractionnel.

\section{Стабильность системы не-Ляпунова частичного порядка с нестационарными временными задержками}

\footnotetext{
В этой статье, критерии стабильности в конечном времени продлены до нелинейных неоднородных возмущённых систем частичного порядка, которые содержат несколько изменяющихся во времени задержек. Получены достаточные условия стабильности для систем частичного порядка с несколькими временными задержками, с использоваными обобщенными и классическими подходами Гронвалла (Gronwall). Численный пример приведён для того, чтобы проиллюстрировать значение полученных результатов.

Ключевые слова: непрерывная система, нелинейная система, система с задержкой, временная задержка, стабильность системы, стабильность не-Ляпунова, стабильность в конечном времени, система дробнго порадка.
} 